\title{
Multiple sclerosis in black South Africans and Zimbabweans
}

\author{
G Dean, A I G Bhigjee, P L A Bill, V Fritz, I C Chikanza, J E P Thomas, L F Levy, \\ D Saffer
}

\begin{abstract}
Multiple sclerosis is rare among the indigenous black people of Africa. The first account of a black patient with multiple sclerosis in South Africa was published as late as 1987. Since then a search to find black patients with multiple sclerosis in Southern Africa has continued. Seven black patients have now been traced in South Africa and five in Zimbabwe in whom a diagnosis of multiple sclerosis can be accepted. Six of the 12 patients became blind, or nearly so, from severe optic neuritis. Multiple sclerosis in these few black patients more often resembled the disorder as it occurs in oriental people than among white people in southern Africa or the black people of North America or the Caribbean.
\end{abstract}

(F Neurol Neurosurg Psychiatry 1994;57:1064-1069)

Medico-Social

Research Board, Dublin, Ireland G Dean

Department of Neurology, Faculty of Medicine, Wentworth Hospital, Durban, South Africa A I G Bhigjee P L A Bill

Medical School, University of Witwatersrand, Johannesburg, South Africa V Fritz

Rheumatology Unit, UMDS, London, England

C Chikanza

Department of

Medicine and of

Neurosurgery,

University of

Zimbabwe, Harare,

Zimbabwe

JE P Thomas

L F Levy

Faculty of Medicine,

University of

Witwatersrand;

Department of

Neurology,

Baragwanath

Hospital, Soweto,

South Africa

D Saffer

Correspondence to:

Dr Geoffrey Dean, PO Box

Dublin 4, Ireland.

Received 16 August 1993

and in final revised form

4 February 1994.

Accepted 18 February 1994 were known to the South African Multiple Sclerosis Society.

In Zimbabwe, one of us (ICC) has collected information about some black patients with multiple sclerosis and further information was obtained from the Zimbabwean Multiple Sclerosis Society and from the patients' doctors. Some of the Zimbabwean patients with multiple sclerosis had also been examined by visiting neurologists from abroad and they agreed with the diagnosis. The death certificates in Zimbabwe were not searched for deaths reported as due to multiple sclerosis.

\section{Case histories \\ SOUTH AFRICA \\ Case 1}

(Wentworth Hospital, Natal) In 1987 Bhigjee $^{12}$ reported a 46 year old Zulu man with a seven year history of progressive weakness of the legs and clumsiness of the right hand, with no remission. There was no family history of a similar illness or history of alcohol abuse or exposure to toxins or trauma.

On examination he had a right internuclear ophthalmoplegia and nystagmus on upward gaze. The optic fundi and pupillary responses were normal. The tendon reflexes were exaggerated, the abdominal responses were absent, and the plantar responses were extensor. There was pronounced ataxia of all four limbs so that he was unable to walk. Oligoclonal bands were found in his CSF. The treponemal antibody absorption test was positive in the blood but negative in the spinal fluid. Tests for cysticercosis were negative. The visual evoked response was delayed in both eyes $(\mathrm{P} 100=121$ and $116 \mathrm{~ms})$. His human leucocyte antigen (HLA) groups were A2, AW30, B7, BW42, DR2, DR3, DQ1, DQ3. MRI showed multiple high intensity lesions in a periventricular distribution. $\mathrm{He}$ is considered to have laboratory supported probable multiple sclerosis by the Poser classification. ${ }^{13} \mathrm{He}$ has been lost to follow up.

\section{Case 2}

(Wentworth Hospital, Natal) This 26 year old man was first seen in 1984 with a three day history of pain, numbness, and weakness in both legs, followed by urinary retention. On examination at that time, his mental state was normal. His fundi were normal and he had nystagmus on looking to the left. $\mathrm{He}$ was dysarthric and had incoordination of both upper limbs. All tendon reflexes were brisk, the abdominal reflexes were absent, and both 
plantar responses were extensor. There was impairment to pinprick and touch sensation in the lower limbs but vibration and joint position sense were normal. The rapid plasma reagin test for syphilis was positive to a dilution $1: 2$ but the corresponding test in the CSF was negative. A myelogram and CT of the brain were normal. An EEC showed occasional bursts of frontal intermittent rhythmic activity. Although the evidence for neurosyphilis was limited he was given a course of penicillin. He improved gradually over a period of five weeks and, at the time of discharge, was able to walk with only mild disability. In 1988 he developed the rapid onset of hoarseness, weakness of the legs and the right arm, and incontinence of urine and faeces. Examination at that time showed a normal mental state, pallor of the left optic disc, mild weakness of abduction of the right eye, horizontal nystagmus, and asymmetric tongue and palatal movements. Tone was increased in all four limbs. The tendon reflexes were brisk bilaterally and both plantar responses were extensor. Touch and pinprick sensation were impaired up to the T12 dermatome. Joint position sense was intact but vibration sense was impaired in the legs. There was incoordination of the arms. A repeat myelogram and CT of the head were normal as were the serum B12 and folic acid. The rapid plasma reagin test in blood was positive for syphilis, but the test was negative in CSF. Antibodies to HTLV1 were not detected in the blood or CSF. A diagnosis of clinically definite multiple sclerosis was made and the patient was treated with prednisone. $\mathrm{He}$ improved to the extent that he could walk between parallel bars. He failed to return for follow up.

\section{Case 3}

(Wentworth Hospital, Natal) This 40 year old woman was first seen in May 1987. She gave a 10 year history of progressive difficulty in walking, disturbance of balance, shaking of the right arm and right leg, and slurred speech. There was no history of exposure to toxins. There was no family history of a similar illness. Examination showed bilateral horizon-
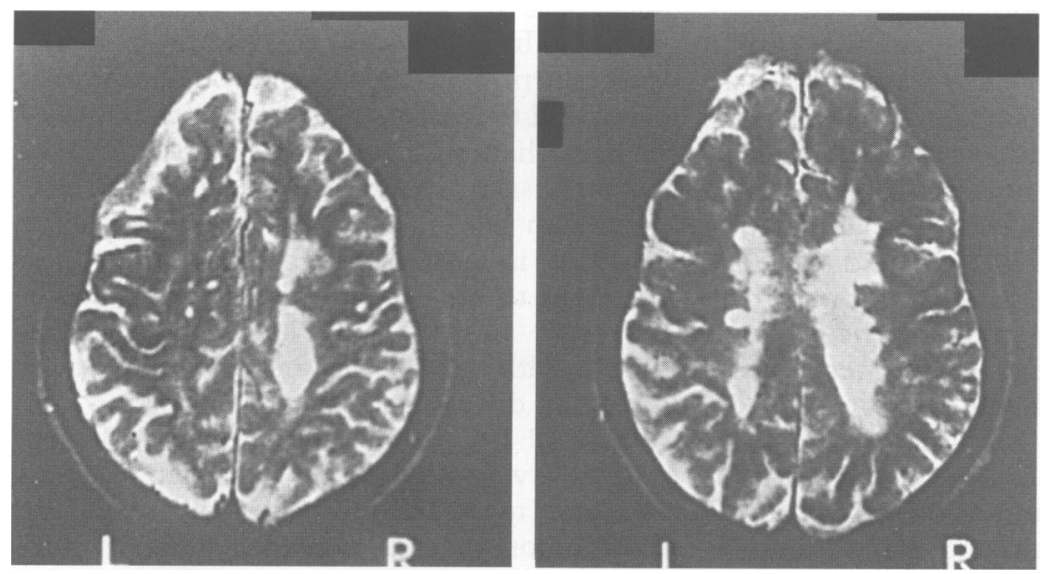

Figure 1 MRI of patient 3. There are extensive white matter lesions in the periventricular and subcortical areas. tal nystagmus and dysarthria. Her visual acuity, the fundi, and cranial nerves were normal. Tone was increased in all four limbs. Power in the arms was normal but it was decreased in the legs. Deep tendon reflexes were brisk, more so on the right than on the left. The abdominal reflexes were absent and both plantar responses were extensor. Touch and pinprick sensation were normal but vibration sense and position sense was absent in the feet. There was bilateral cerebellar ataxia, more pronounced on the right side. A myelogram showed no abnormality. The CSF was acellular and had a protein concentration of $0.22 \mathrm{~g} / 1$. The blood rapid plasma reagin test for syphilis was negative.

She was readmitted in May 1989 with a rapid decrease in visual acuity in the left eye and a similar, but more gradual, failure of vision in the right eye. She also complained of urgency and incontinence of urine. Examination confirmed progression of cerebellar and pyramidal dysfunction and she also had bilateral internuclear ophthalmoplegia. She had optic atrophy of the left eye and mild disc pallor of the right. The visual acuity of the left eye was reduced to an ability to count fingers, and was $6 / 36$ in the right eye. She was confined to a wheelchair. A repeat CT scan of the brain, after double dose contrast, was unremarkable except for generalised atrophy. The right visual evoked response was delayed $(\mathrm{P} 100=135 \mathrm{~ms})$. No discernible waveform was noticeable from the left eye. The late waves of the brainstem auditory evoked responses were absent on the right and delayed on the left. The median somatosensory evoked response was normal on the right and delayed on the left $(\mathrm{N} 20=25.6 \mathrm{~ms})$. The CSF was, again, acellular but the IgG index was raised $(1 \cdot 1$; normal $<0.07)$. The HLA profile was A26, A28, B7, B14, DR2, DR53, and DQ1. Antibody tests for HIV and HTLV1 in the serum gave negative results. MRI (fig 1) showed extensive white matter lesions in the periventricular and subcortical areas, as well as in the brainstem and cerebellum. She was considered to have clinically definite multiple sclerosis. She improved with treatment with azathioprine. In subsequent admissions there was considerable neurological deterioration and she had repeated urinary tract infections. She died in September 1990 and there was no postmortem examination.

\section{Case 4}

(Wentworth Hospital, Natal) This 36 year old man presented in 1990 with a three year history of progressive weakness of both legs, the right greater than left. He also developed urgency of micturition and poor vision and incoordination of his right arm. There was no history of exposure to toxins or substance abuse, nor a family history of any similar problem. On examination his mental state seemed normal. There was pallor of both optic discs. There was bilateral and rotatory nystagmus. He had spastic paraparesis, generalised hyper-reflexia, and a bilateral extensor plantar response. There was ataxia of both 

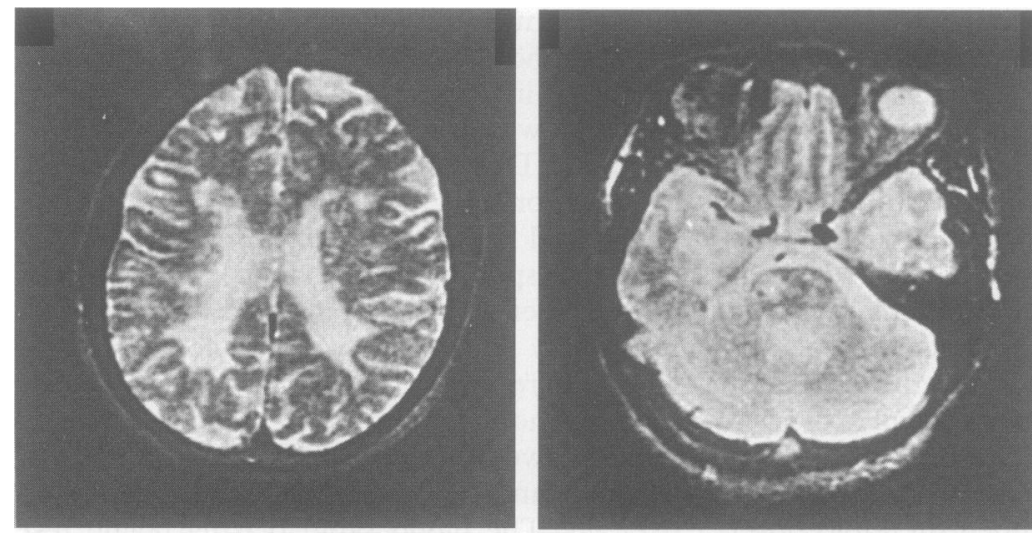

Figure 2 MRI of patient 4. There are extensive lesions-both focal and confluent.

upper limbs. Vibration sense was absent at the ankle and joint sense was impaired. $\mathrm{He}$ walked with a walking stick. The CSF IgG was $0.2 \mathrm{~g} / 1$. MRI (fig 2) showed extensive lesions typical of multiple sclerosis in the cerebral hemispheres and brain stem, both focal and confluent in nature. The right median somatosensory response showed an $\mathbf{N}_{20}$ latency of $24.8 \mathrm{~ms}$ and the left a latency of $23.2 \mathrm{~ms}$. Other investigations, including tests for syphilis, HIV, and HTLV1 were negative. $\mathrm{He}$ was considered to have laboratory supported probable multiple sclerosis and was treated with prednisone. He did not return for follow up.

\section{Case 5}

(Hillbrow Hospital, Transvaal) This 47 year old woman was admitted to hospital in April 1987 with rapidly diminishing vision and pain in the left eye. After three days she became blind in this eye despite treatment with prednisone. In August 1987 she developed pain and diminished vision in the right eye which also became blind after a few days. In August 1988 she complained of a burning pain in the legs, which slowly resolved. In August 1989 she developed weakness in her left arm and in March 1991 she returned to hospital with pronounced weakness in the left arm and some weakness in the right arm. She also complained of urinary and faecal incontinence and loss of sensation in both legs.

On examination in July 1991, she was blind and confined to a wheelchair. She had no speech defects. There was bilateral optic atrophy and no pupillary reaction to light. There were no involuntary muscle movements. Tone was increased in the left arm and right leg and decreased in the right arm and left leg. Both knee jerk responses were absent and the left ankle jerk was also absent. The triceps and biceps reflexes were normal. Plantar responses could not be obtained. There was diminished sensation to touch and pinprick on the left side. She had loss of position and vibration sense in both legs. It was not possible to test for ataxia. The myelogram and CT were normal. An HIV test and a Wasserman reaction were negative. There was increased IgG in the
CSF. Tests for cysticercosis were negative. No improvement was noted throughout the patient's stay in hospital and she remained tetraplegic. She was diagnosed as having clinically definite multiple sclerosis. She died in September 1991 and there was no postmortem examination.

\section{Case 6}

(Johannesburg Hospital, Transvaal) This 42 year old Shangaan woman first noticed weakness and unsteadiness in her legs in 1989. On examination, in January and July 1990, there was nystagmus on upward gaze, she could not abduct the right eye, and there was left upper motor neuron facial palsy. She had a spastic tetraparesis, most pronounced in the legs. All her reflexes were brisk and she had bilateral ankle clonus and extensor Babinski's responses. There was truncal ataxia with an abnormal finger to nose test. Her myelogram was normal, and a Wasserman reaction and HIV antibody tests were negative. There was a prolonged latency in both her visual and auditory responses. Her HLA analysis was A30, B13, CW2, W6, DR1·5(11), DRW52, DQW1, and DQW3. MRI showed the classic periventricular lesions that are seen in multiple sclerosis and there were also lesions of the cerebellum, brainstem, and corpus callosum. She was given methyl prednisone with no improvement in her condition. When she was seen again, in May and October 1991, there had been no remission. There was left sided optic atrophy. She had a right internuclear ophthalmoplegia. There was left upper motor neuron facial weakness and spastic tetraparesis, mainly affecting the lower limbs, so that she could not walk. Touch sensation was diminished distally in the lower limbs. She had a truncal ataxia, with abnormal finger to nose tests and bilateral dysdiadochokinesia. Oligoclonal bands were present in the CSF. She was diagnosed as having clinically definite multiple sclerosis. She died in January 1992 and there was no postmortem examination.

\section{Case 7}

(Groote Schuur Hospital, Capetown, and Johannesburg Hospital, Transvaal) This woman was born in 1943 in the Transkei. Her mother was a Swazi and her father a Zhosa. There was no known history of inter-racial marriage. In 1974, at the age of 31, she developed blurring of vision in the left eye that responded to treatment with steroids. In 1979 she again had blurring of vision in the left eye and the vision recovered once again on treatment with steroids. In 1981 she developed weakness in both legs, which improved on treatment to the extent that she was ambulatory with aid. In 1987 she had a further relapse with severe weakness in both lower limbs that did not improve.

In April 1990, she had acute onset of spasticity and weakness of the right arm and was admitted to hospital in Johannesburg. On examination at that time, her mental functions and speech were normal. There was no nystagmus. Both lower limbs were totally 
paralysed and flaccid. There was weakness and spasticity of both upper limbs, most pronounced in the right arm. She was incontinent of urine. There was loss of touch, pain, position, and vibration sense from the level of $\mathrm{T} 2$. It was difficult to assess coordination although she had an element of intention tremor in her arms. The visually evoked response was delayed in both eyes. Brain MRI showed lesions scattered throughout the periventricular area, typical of those seen in multiple sclerosis. Her CSF was normal and Wasserman and fluorescent treponemal antibody tests were negative. She was considered to have clinically definite multiple sclerosis. She died in 1992 and there was no postmortem examination.

The neurology unit at Baragwanath Hospital, Soweto, Johannesburg, has seen five patients during the past 20 years-two of them in the past five years - in whom a diagnosis of multiple sclerosis was seriously considered. The two most recent patients had internuclear ophthalmoplegia and unilateral optic atrophy and one had evidence of pyramidal tract and posterior column involvement and a delayed visual evoked response. In both of the recent patients oligoclonal bands were present on analysis of CSF. All five patients had, however, coexisting infections of the nervous system, in three the blood and CSF examination showed antibodies for treponema infection; in the fourth patient there were both antibodies for syphilis and HTLV1 infection in the CSF and the fifth patient had raised cysticercosis antibodies. The primary infections were treated with some clinical improvement. None of the five patients returned for follow up.

Two other female black South Africans were reported as dying from multiple sclerosis on their death certificates, but good histories for these patients have not so far been obtained.

\section{ZIMBABWE}

Case 8

(Harare and Parirenyatwa Central Hospitals, Harare) In 1987, this 47 year old nursing sister developed weakness and loss of touch sensation in her left arm. This recovered spontaneously. In February 1988, she developed pain and stiffness in her neck, followed by numbness of the right arm and on the right side of the body and also, later, some weakness of the left arm and leg and loss of touch sensation. On examination at that time she had nystagmus, scanning speech, dysdiadochokinesis, and intention tremor and there was profound proprioceptive loss in her left arm. Improvement followed steroid treatment.

In July 1990, she developed weakness and numbness of her arms and legs and was seen by a visiting neurologist, $\mathrm{Dr} \mathrm{D}$ Leary, who agreed that she had multiple sclerosis and she was treated with methyl prednisolone infusion. This resulted in some improvement. In October 1990, she developed blurring of vision in the right eye and pronounced optic atrophy was noted. In July 1991, she was readmitted to hospital with a relapse of weakness in the left arm and leg, again she improved on treatment with prednisolone. A further relapse occurred in July 1992. She is considered to have clinically definite multiple sclerosis.

\section{Case 9}

(Harare and Parirenyatwa Central Hospitals, Harare) In 1988, an 18 year old man developed blurred vision in his right eye, numbness in his legs and hands and, later, weakness of the hands. He was treated with prednisolone and recovered. He was seen by one of us (LFL) in December 1988, who found that vision had almost returned to normal in the right eye. At that time he was areflexic in the arms and he had poor temperature and vibration sense in the lower limbs. There was slight ataxia on the right finger to nose test and on alternating movements of the hand. Lhermitte's sign was positive. A CT was normal and lumbar puncture showed normal protein and a negative Pandy's test. The patient improved on treatment with prednisolone.

In December 1989, he developed spasms of his left hand; again he was given prednisolone with some improvement. In June 1991 he had a further relapse with numbness in his legs and abdomen. In June 1992 he developed pain and severely impaired vision in his right eye and difficulty in starting micturition. $\mathrm{He}$ improved with prednisolone treatment. He was a student at the University in 1993, and, except for his visual symptoms, was reasonably well. $\mathrm{He}$ is considered to have clinically definite multiple sclerosis.

\section{Case 10}

(Mpilo Hospital, Bulawayo) In 1977 a 22 year old man noticed that he could not control the use of his left arm. When he was examined at that time by Dr I Rachman he had nystagmus and ataxia of the left upper limb and there was pallor of the left optic disc. He was admitted to hospital where he developed mild right facial weakness and difficulty with coughing and swallowing. Ataxia in the left arm increased and the left leg was also unsteady. He gradually improved but in 1978 he developed poor vision in the left eye and was found to have a large central scotoma. He recalled two previous episodes of impaired vision in the left eye in March and September of 1977. $\mathrm{He}$ was considered to have multiple sclerosis. In 1978 he had an epileptic seizure and later admitted that he had had a previous seizure in August 1976. During 1988 and 1989 he was attended by one of us (LFL). At this time he had a loss of balance and was unable to walk without using a stick. He was totally blind in his right eye and had poor vision in the left eye. He is considered to have clinically definite multiple sclerosis.

\section{Case 11}

(Harare and Parirenyatwa Central Hospitals, Harare) This 29 year old woman lost the vision in her left eye in 1974 and in her right 
eye in 1975. In 1983, she developed hemiparesis of the right side, which recovered over one month. At that time she was noted to have bilateral optic atrophy, worse on the right side. A cranial angiogram showed no pathology. Routine testing of her CSF was normal.

She was seen again in 1986, when she had bilateral optic atrophy, weakness of both legs, extensor Babinski's responses, and oligoclonal bands in her CSF. By June 1989 she spent most of the day in a wheelchair. She complained of painful flexor spasms in her legs and constipation. By June 1.992 she had spastic tetraplegia.

By September 1992 power in her legs had improved but she was totally blind. Her mental acuity remains undiminished and she enjoys books provided by tape aids for the blind. She is considered to have clinically definite multiple sclerosis.

\section{Case 12}

(Harare and Parirenyatwa Central Hospitals, Harare) In 1988, a 40 year old man presented with difficulty with balance. $\mathrm{He}$ also complained of pain in his knees and forgetfulness. In 1990, he developed almost total loss of vision in the right eye and, six months later, blurring of vision in the left eye. By then the vision in the right eye had partially recovered. $\mathrm{He}$ also complained of a strange sensation in his feet. He had no bowel or bladder symptoms. On examination his gait was unsteady and his speech was slurred. There was ataxia of both arms and legs. The limb reflexes were hyperactive and the Babinski's responses were extensor. His vision was very poor so that he could just see his fingers. From his history and the clinical findings, he is considered to have clinically definite multiple sclerosis.

All of the five Zimbabwean patients with multiple sclerosis maintain that they had no white ancestors. A further young black man from Zimbabwe attended the University of Pennsylvania as a medical student in the early 1970 s and was diagnosed there as having multiple sclerosis.

\section{Discussion}

Our case series is strong evidence that multiple sclerosis does occur in black Southern Africans, although there is, as yet, no postmortem confirmation. Multiple sclerosis is, however, very rare among them. There seems to be a gradient in Southern Africa from a relatively high prevalence of multiple sclerosis in white immigrants from Europe to a lower prevalence in white English speaking African born, a somewhat lower prevalence in white Afrikaans speaking African born, a low prevalence among the coloured and Asian population (Indian and Chinese) and, lastly, a very low multiple sclerosis prevalence among the black population. 8914

Devic's syndrome, ${ }^{15}$ or neuromyelitis optica, seems to be as common among the black population in Africa, ${ }^{371011}$ in comparison with multiple sclerosis, as it is in Japan and China. ${ }^{1617}$ In the present report, six of the 12 black patients considered to have multiple sclerosis became blind, or nearly blind, an uncommon occurrence in multiple sclerosis in Europe or North America or among white South Africans. By contrast with the black patients with multiple sclerosis in Africa, the black people of North America, or the AfroCaribbean people who have immigrated to England and who have a largely black ancestry, have a similar pattern of multiple sclerosis to that which occurs among the white population. ${ }^{18-21}$

Seven black immigrants to England from Africa ${ }^{1819}$ are known to have been diagnosed as having multiple sclerosis, one of whom became totally blind. One has died and there was a postmortem examination. She was an 11 year old girl when, in 1970, she developed an insidious right hemiparesis over a period of two months and unsteadiness of both arms. She was investigated at the National Hospital, Queen Square, by Dr Michael Kremer, who considered that she had multiple sclerosis. She made a complete recovery. She had a relapse in July 1971 and again recovered. In July 1973 , she was readmitted to the National Hospital because of seizures.

Subsequently she developed abdominal pain, a macular skin rash, jaundice, haematemesis, and died. Necropsy showed massive hepatic necrosis with many petechial haemorrhages. The brain was atrophic but was too poorly preserved for reliable histological assessment.

Multiple sclerosis is uncommon not only among indigenous black people of Africa, but also among the semitic people of Northern Africa and of Malta. There is a steep fall in multiple sclerosis prevalence from Sicily, where the prevalence is around 50 to 60 per $100000,{ }^{22}$ to the nearby island of Malta where, in a population of 350000 people, only 13 patients with multiple sclerosis could be found and one man, totally blind, who had been diagnosed as having Devic's syndrome. ${ }^{23}$ The pattern of multiple sclerosis prevalence seems to change from Southern Europe, where it is relatively high, to Malta, where it is low, and to the indigenous people of Africa. Further studies of the genetic and environmental factors that are responsible for the difference in the pattern of multiple sclerosis in Africa compared with Europe and Northern America should shed light on the cause of this strange disease.

The South African Bureau of Statistics, Pretoria, provided copies of the death certificates of the South African black patients in which multiple sclerosis was mentioned. Mrs Lorn Robinson and the Multiple Sclerosis Society of Zimbabwe assisted us in obtaining the histories of the Zimbabwe patients. Professor Ian McDonald of the National Hospital, Queen Square, advised us.

1 Goldstein B. Two cases of disseminated sclerosis in African natives. East Afr Med $\mathcal{F}_{1946 ; 23: 170-3}$ 2 Foster RM, Harries JR. Multiple sclerosis in the African $B M \mathcal{F}$ 1970;3:628

3 Adam AM. Multiple sclerosis: epidemic in Kenya. East Afr Med $\mathcal{f} 1989 ; 66: 503-6$.

4 Kanyerezi BR, Kiire CF, Obace A. Multiple sclerosis in Mulago Hospital, Uganda. East Afr Med $\mathcal{f} 1980 ; 57$. 262-6. 
5 Mbonda E, Larnaout A, Maertens A, et al. Multiple sclerosis in a black Cameroonian woman. Acta Neurol Belg 1990;90:218-22.

6 Haimanot R. MS - a case report on an Ethiopian. Ethiop Med F 1985;23:27.

7 Vigneront F. La sclerose en plaques au Senegal. Nancy, France: University of Nancy, 1976 (Thesis).

8 Dean G. Disseminated sclerosis in South Africa. BMF 1949;1:842-4.

9 Dean G. Annual incidence, prevalence, and mortality of multiple sclerosis in white South African-born and in multiple sclerosis in white South African-born and in
white immigrants to South Africa. $B M 7$ 1967;2:724-30.

$10 \mathrm{Hift}$ W, Moodley T. A possible case of neuromyelitis optica in a Bantu patient. $S$ Afr Med $\mathcal{F} 1973 ; 47$ :987-8.

11 Cosnett JE. Multiple sclerosis and neuromyelitis optica. $S$ Afr Med $\mathcal{1}$ 1981;60:249-51.

12 Bhigjee AI. Multiple sclerosis in a black patient. A case report. $S$ Afr Med $\mathcal{f}$ 1987;72:873-5.

13 Poser CM, Paty DW, Scheinberg L, et al. New diagnostic criteria for multiple sclerosis: guidelines for research protocols. Ann Neurol 1983;13:3:227-31.

14 Dean G, Elian M. Motor neuron disease and multiple sclerosis in Australia, New Zealand and South Africa compared with England and W/ales. 7 Neurol Neurosurg Psychiatry 1993;56:633-7.

15 Devic M. Myelite subaigue compliquée de neurite optique. Le Bulletin Medical 1894;8:1033-4.
16 Kuroiwa Y, Kurland LT. Multiple sclerosis East and West. Proceedings of the Asian multiple sclerosis workshop, Kyoto, 1981. Kyushu University Press, 1982.

17 Arnason BGW, Davis FA, Dean G, Kelly R, Sever JL, Waksman BH. Round the world from our correspondents: China-demyelinating diseases. Lancet 1982;1: 734

18 Dean G, McLoughlin H, Brady R, Adelstein AM, TallettWilliams J. Multiple sclerosis among immigrants in Greater London. $B M 7$ 1976;1:861-4.

19 Dean G, Brady R, McLoughlin H, Elian M, Adelstein AM. Motor neurone disease and multiple sclerosis AM. Motor neurone disease and multiple sclerosis
among immigrants in Britain. British fournal of among immigrants in Britain. British

20 Elian M, Dean G. Multiple sclerosis among the United Kingdom-born children of immigrants from the West Indies. F Neurol Neurosurg Psychiatry 1987;50:327-32.

21 Elian M, Nightingale S, Dean G. Multiple sclerosis among United Kingdom-born children of immigrants from the Indian Subcontinent, Africa, and the West Indies. I Neurol Neurosurg Psychiatry 1990;53:906-11.

22 Dean G, Grimaldi G, Kelly R, Karhausen L. Multiple sclerosis in southern Europe I- prevalence in Sicily in 1975. F Epidemiol Community Health 1979;33:107-10.

23 Vassalo L, Eliam M, Dean G. Multiple sclerosis in southern Europe II: prevalence in Malta in 1978. F Epidemiol Community Health 1979;33:111-3.

\section{NEUROLOGY IN LITERATURE}

\section{Neuralgia}

Neuralgia covers a multitude of disorders in literature as well as in neurology.

Not only novelists have been confused by the term tic douloureux and whether it can be simply regarded as a synonym for trigeminal neuralgia. Early accounts of trigeminal neuralgia stressed the occurrence of reflex muscle spasms during the painful paroxysm; hence the alternative title for the condition. Gowers called these movements tic convulsif, ignoring the fact that French neurologists had applied that term to cases of hemifacial spasm.

Mr Weller's condition sounds in fact, more like hemifacial spasm than trigeminal neuralgia whereas the neuralgic pains described by Joyce, Saki, and Edith Wharton are insufficiently detailed to be confident that they refer to trigeminal neuralgia. Dostoyevsky places a paroxysm of pain in the temple, not a particularly common site for the condition, and Elizabeth Gaskell's coupling the problem with sickness and fever suggests that she was dealing with a different disorder. The resistance of the pain to even potent analgesics was clearly known to Sheridan Le Fanu, and its severity to Herman Melville. The last word belongs to Proust. Charcot, incidentally, receives frequent mention in Remembrance of things past.

Charles Dickens, 1836-7, The Pickwick papers $\mathrm{Mr}$ Weller communicated this secret with great glee, and winked so indefatigably after doing so, that Sam began to think he must have got the tic douloureux in his right eye-lid.

Herman Melville, 1851, Moby Dick

"It's a kind of tic-dolly-row they say-worse nor a toothache. Well, well; I don't know what it is, but the Lord keep me from catching it".

Elizabeth Gaskell, 1857, The life of Charlotte Bronte Martha has suffered from tic-douloureux, with sickness and fever, just like you.

I Sheridan Le Fanu, 1864, Uncle Silas

To this hour I cannot say what was the nature of those periodical seizures. I have often spoken to medical men about them, since, but never could learn that excessive use of opium could altogether account for them. It was, I believe, certain, however, that he did use that drug in startling quantities. It was, indeed, something of a topic of complaint with him that his neuralgia imposed this sad necessity upon him.

F Dostoyevsky, 1871, The devils

Mrs Stavrogin raised her head slightly and pressed the fingers of her right hand gingerly to her right temple, evidently feeling an acute twinge of pain there.

Edith Wharton, 1912, The reef

"Oh, don't-I hate the lights!" Owen exclaimed, catching her by the wrist and pushing her back into the seat. He gave a nervous laugh and added: "I'm half blind with neuralgia. I suppose it's this beastly rain."

Marcel Proust, 1921-2, Remembrance of things past: cities of the plain

Then, when Cottard was scarcely known to the public, if you spoke to $M$ Verdurin of his wife's facial neuralgia, "There is nothing to be done," he would say, with the naive complacency of people who assume that anyone whom they know must be famous, and that everybody knows the name of their daughter's singing-teacher. "If she had an ordinary doctor, one might look for a second opinion, but when that doctor is called Cottard" (a name which he pronounced as though it were Bouchard or Charcot) "one simply has to bow to the inevitable."

fames foyce, 1922, Ulysses

An old man widower, unkempt hair, in bed, with head covered, sighing: an infirm dog, Athos: Aconite, resorted to by increasing doses of grains and scruples as a palliative of recrudescent neuralgia ...

Saki, The toys of peace

Miss Louise has been upstairs all the afternoon, Ma'am, reading to the second kitchenmaid, who has the neuralgia.

G D PERKIN

Regional Neurosciences Centre, Charing Cross Hospital, London W6 8RF, UK 